doi:10.13108/2016-8-2-104

\title{
ON THE EXPANSION OF A MEROMORPHIC FUNCTION IN PARTIAL FRACTIONS
}

\section{L.S. MAERGOIZ}

\begin{abstract}
The paper is devoted to the expansion in partial fractions for a meromorphic function of one complex variable. It contains the results by the author on representing a meromorphic function as a sum of an entire function and the principal parts in its Laurent expansion at its poles.

Keywords. Expansion in partial fractions, reciprocal of entire function, meromorphic function, proximate order, indicator.
\end{abstract}

Mathematics Subject Classification: 30A20

Dedicated to Igor Fedorovich

Krasichkov-Ternovskii on the occasion of his 80th birthday

\section{INTRODUCTON}

We choose a meromorphic function $\Phi$ in $\mathbb{C}$. Let $\Lambda=\left\{b_{k}\right\}_{1}^{\infty}$ be the sequence of its poles taken in the ascending order of their absolute values and tending to $\infty$. Let

$$
\left\{\Phi_{k}(z)=\sum_{n=1}^{N_{k}} \frac{A_{k, n}}{\left(z-b_{k}\right)^{n}}: k=1,2, \ldots\right\}
$$

be the sequence of principal parts of the Laurent expansion for function $\Phi$ at the poles. The following assertion was proved by Mittag-Leffler (see, e.g. [1, Ch. 7]).

In the notation of (1.1) any meromorphic function $\Phi$ in $\mathbb{C}$ admits an expansion

$$
\Phi(z)=\sum_{k=1}^{\infty}\left[\Phi_{k}(z)+P_{k}(z)\right]+\alpha(z), \quad z \in \mathbb{C} \backslash \Lambda,
$$

where $\alpha$ is an entire function and $\left\{P_{k}(z)\right\}_{1}^{\infty}$ is a sequence of polynomials.

This paper is devoted to description of some conditions under which

$$
P_{k}(z) \equiv 0, z \in \mathbb{C} ; k \in \mathbb{N}
$$

in formula (1.2). Preliminary results of this paper were announced in [2], where, moreover, there was a short survey of studies devoted to partial fractions expansion for meromorphic function of one complex variable (results by M.G. Krein [3, Lect. 16], M.V. Keldysh and I.V. Ostrovskii [4, Ch. 5], I.V. Ostrovskii [5]). In addition, we mention the review by A.A. Gol'dberg [6], paper by J.Clunie, A. Eremenko, J. Rossi [7], V.B. Sherstyukov [8], [9].

\footnotetext{
Л.С. МАЕРГОЙЗ, О РАЗЛОЖЕНИИ МЕРОМОРФНОЙ ФУНКЦИИ НА ПРОСТЕЙШИЕ ДРОБИ.

(C) МАЕРГОЙз Л.С. 2016.

Поступила 21 ноября 2015 г.
} 


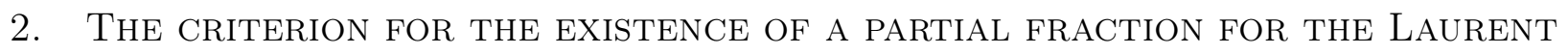
EXPANSION OF A MEROMORPHIC FUNCTION

Definition 1. ([10, Def. 6.5]). We shall say that a meromorphic function $\Phi$ can be expanded into partial fractions if, in the notation of the Mittag-Leffler Theorem, we have

$$
\Phi(z)=\alpha(z)+\phi(z), \quad z \in \mathbb{C} \backslash \Lambda, \quad \phi(z)=\lim _{R \rightarrow \infty} \Phi(z ; R) ; \Phi(z ; R)=\sum_{\left|b_{k}\right|<R} \Phi_{k}(z)
$$

where $\alpha$ is an entire function and series $\phi(z)$ converges uniformly on every compact subset of C. It means that for any $R>0$ the series

$$
\phi(z)-\Phi(z ; R)=\sum_{\left|b_{k}\right| \geqslant R} \Phi_{k}(z)
$$

converges uniformly on every compact subset of $S_{R}=\{z \in \mathbb{C}:|z|<R\}$ (see [1, Ch. 7]).

The simplest example of such function is the Gamma-function. It can be represented as (see, e.g. [1, Ch. 7])

$$
\Gamma(z)=\sum_{k=0}^{\infty} \frac{(-1)^{k}}{k !(z+k)}+\alpha(z), \quad \alpha(z)=\int_{1}^{\infty} e^{-t} t^{z-1} d t .
$$

Proposition 1. Let, in the notation of (1.1) and Definition 1, $\left\{R_{n}\right\}_{1}^{\infty}$ be the increasing sequence of radii of all circumferences centered at the origin and containing the poles of $\Phi$. A meromorphic function $\Phi$ can be expanded into partial fractions if there exists the limit in (2.1) at least for one increasing sequence of positive numbers $\left\{r_{n}\right\}_{1}^{\infty}$ such that for some $k \in \mathbb{N}$ we have

$$
R_{k+n}<r_{n}<R_{k+n+1} \quad \forall n \in \mathbb{N} .
$$

We shall make use the following convergence criterion for the series of principal parts in the Laurent expansion of a meromorphic function.

Theorem 1 ([10, Thm. 6.4]). In the notation of the Mittag-Leffler theorem the series

$$
\sum_{k=1}^{\infty} \Phi_{k}(z):=\lim _{R \rightarrow \infty} \sum_{\left|b_{k}\right|<R} \Phi_{k}(z)
$$

(see (1.1)) converges for all $z \in \mathbb{C} \backslash \Lambda$ if and only if the series

$$
R(z):=\lim _{R \rightarrow \infty} \sum_{\left|b_{k}\right|<R} \operatorname{res}_{w=b_{k}} \Psi(w, z), z \in \mathbb{C} \backslash \Lambda ; \Psi(w, z)=\frac{\Phi(w)}{w-z}
$$

converges. Moreover,

$$
\Phi_{k}(z)=-\underset{w=b_{k}}{\operatorname{res}} \Psi(w, z), z \in \mathbb{C} \backslash \Lambda, k \in \mathbb{N} ; R(z)=-\sum_{k=1}^{\infty} \Phi_{k}(z) .
$$

If series $R(z)$ converges uniformly on every compact subset of $\mathbb{C}$, then

$$
\alpha(z)=\Phi(z)-\sum_{k=1}^{\infty} \Phi_{k}(z)
$$

is an entire function equal to the total sum of residues of $\Psi(w, z)$ in variable $w$. 


\section{ENTIRE FUnCTIONS OF COMPLETELY REGULAR GROWTH}

We recall some concepts in the theory of entire functions [11.

Let $E$ be a measurable set of positive numbers. Denote by $m$ the Lebesgue measure on $(0, \infty)$. Set $E$ is called a set of relative measure zero or a $E^{0}$-set if

$$
\lim _{r \rightarrow \infty} r^{-1} m[E \cap(0, r)]=0 .
$$

Definition 2. ([11, Ch. 3]). Let $A[\rho(r)]$ be the class of entire function $f$ of finite order $\rho>0$ and of normal type with respect to proximate order $\rho(r), \rho(r) \rightarrow \rho$. A function $f \in A[\rho(r)]$ is called a function of completely regular growth $(C R G)$ on the ray $\arg z=\theta$, where $\theta \in \mathbb{R}$, if the limit

$$
h_{f}(\theta)=\lim _{r \rightarrow \infty} r^{-\rho(r)} \ln \left|f\left(r e^{i \theta}\right)\right|
$$

exists along $r \in \mathbb{R}_{+} \backslash E$ for some $E^{0}$-set $E$. Here $h_{f}$ is the generalized indicator of $f$. If in (3.1) the convergence to the limit as $r \notin E$ is uniform in the variable $\theta \in \mathbb{R}$ and $E$ is a $E^{0}$-set independent of $\theta$, then $f$ is called an entire function of completely regular growth (CRG-function).

We shall need some properties of CRG-functions.

Property 1. In the above notation, there exists an increasing sequence of positive numbers $\mathfrak{r}=\left\{r_{n}\right\}_{1}^{\infty}$ such that

$$
h_{f}(\theta)=\lim _{r \rightarrow \infty} r_{n}^{-\rho\left(r_{n}\right)} \ln \left|f\left(r_{n} e^{i \theta}\right)\right|,
$$

and convergence in (3.2) is uniform in $\theta \in \mathbb{R}$. Moreover, sequence $\mathfrak{r}$ satisfies inequality (2.2), where $\left\{R_{n}\right\}_{1}^{\infty}$ is the increasing sequence of radii of all circumferences centered at the origin and containing the zeroes of $f$.

Property 2. Let $f \in A[\rho(r)]$ be a CRG-function such that there are no zeroes of $f$ in an angle $\Omega=\{w \in \mathbb{C}: \gamma<\arg w<\tau\}$. Then the generalized indicator

$$
h_{f}(\theta)=A_{f} \cos \rho \theta+B_{f} \sin \rho \theta, \quad \theta \in(\gamma, \tau), \quad A_{f}, B_{f} \in \mathbb{R}
$$

of $f$ is $\rho$-trigonometrical one on the interval $(\gamma, \tau)$, and the limit exists in (3.1) along $r \in \mathbb{R}_{+}$ for every $\theta \in(\gamma, \tau)$. Moreover, convergence is uniform in any segment $\Delta \subset(\gamma, \tau)$.

This statement is a corollary of the results by V. Azarin [12, Thms. 3.2.7.3, 5.6.1.1] and the properties of harmonic functions [13, Supplement].

\section{Applications of Theorem 1}

We apply the criterion (see Theorem 1) to the partial fraction expansion of a meromorphic function of the form $1 / g$, where $g \in A[\rho(r)]$ is an entire function of CRG. set

Assume that $\Lambda_{g}=\left\{b_{k}\right\}$, where $\Lambda_{g}$ is the set of all zeroes of $g$. Let $F_{g}$ be the closure of the

$$
\left\{\exp \left\{i \arg b_{k}\right\}: \quad b_{k} \in \Lambda_{g} \backslash\{0\}\right\} \subset F_{g} .
$$

Theorem 2. Fix a connected component

$$
B_{g}=\left\{e^{i \theta}: \theta \in(\gamma, \beta)\right\}
$$

of the set $\left\{e^{i \theta} \in \Gamma_{1}: h_{g}(\theta)>0\right\}$, where $\Gamma_{1}=\{z \in \mathbb{C}:|z|=1\}$, and $h_{g}$ is the generalized indicator of $g$. Assume that (see (4.1), (4.2)) $F_{g} \subset B_{g}$. Then the function $\Phi=1 / g$ can be expanded into partial fractions (see Definition 1). Moreover, if $B_{g}=\Gamma_{1}$, then $\alpha(z) \equiv 0$ in the expansion (2.2) for $\Phi$. 
Proof. We check the assumptions of Theorem 1. Suppose that $K$ is an arbitrary compact subset in $\mathbb{C}$. Let $S_{R}=\{z \in \mathbb{C}:|z|<R\}$ be a circle such that $K \subset S_{R}$.

1. Assume that $B_{g} \neq \Gamma_{1}$. Then in the notation of formula (3.1) we have $\beta-\alpha<2 \pi$. Let $[a, b] \subset(\gamma, \beta)$ be the smallest segment such that $F_{g} \subset\left\{e^{i \theta}: \theta \in[a, b]\right\}$. Fix any numbers $c, d$ with the property $c \in(\gamma, a), d \in(b, \beta)$. Let $l:=l(R, c, d)$ be the contour formed by the rays $L_{c}, L_{d}$, where

$$
L_{x}=\{w \in \mathbb{C},|w| \geqslant R, \arg w=x,\}
$$

and the circular arc

$$
l:=l(R, c, d)=\{w \in \mathbb{C},|w|=R, \arg w=\theta \in[d, c+2 \pi]\}
$$

oriented in the growth direction for $\arg w$. We consider the integral

$$
\alpha(z)=\frac{1}{2 \pi i} \int_{L} \Psi(w, z) d w, \quad z \in K
$$

where

$$
\Psi(w, z)=\frac{\Phi(w)}{w-z}, \quad \Phi=g^{-1} .
$$

We take a small number $\varepsilon>0$ such that $\varepsilon<\min \left\{h_{g}(c), h_{g}(d)\right\}$, where $h_{g}$ is the generalized indicator of $g$. Recalling that $g$ is a CRG-function of $A[\rho(r)]$ and taking into consideration that there is no zeroes of $g$ inside the angle $\{w \in \mathbb{C}: b \leqslant \arg w \leqslant a+2 \pi\}$, we find:

$$
\ln \left|g\left(r e^{i \theta}\right)\right|>V(r)\left[h_{g}(\theta)-\varepsilon\right], \quad r>R_{\varepsilon}, \theta=c, d ; V(r)=r^{\rho(r)}
$$

for some $R_{\varepsilon}>0$. By Property 2, letting $\theta=\arg z$, we obtain:

$$
\ln \left|g\left(r e^{i \theta}\right)\right|=V(r) h_{f}(\theta)[1+o(1)], \quad \theta \in(b, a+2 \pi),
$$

as $r \rightarrow \infty$. Furthermore, this asymptotic estimate is uniform in variable $\theta \in[d, c+2 \pi] \subset$ $(b, a+2 \pi)$. Now by (4.3)-(4.6) we can choose $R>R_{\varepsilon}$ such that integral $\alpha(z)$ converges uniformly in variable $z \in K$. By (4.4) we conclude that integral $\alpha(z)$ converges uniformly in the variable $z \in K$. Since $R=R(K)>0$ is an arbitrary fixed number, $\alpha(z)$ is an entire function.

We denote $m=\min \left\{h_{g}(\theta): \theta \in[c, d]\right\}$. Since $[c, d] \subset(\gamma, \beta)$, by (4.2) we have $m>0$. There exists a sequence $\mathfrak{r}=\mathfrak{r}(g)$ satisfying conditions mentioned in Property 1. Therefore, for every $\varepsilon \in(0, m)$ we get

$$
\ln \left|g\left(r_{n} e^{i \theta}\right)\right|>V\left(r_{n}\right)\left(h_{g}(\theta)-\varepsilon\right), n>n_{0}(\varepsilon) \quad \forall \theta \in[c, d] .
$$

Without loss of generality we suppose that $r_{n}>R$ as $n>n_{0}$. Estimate (4.7) implies that, in the notation of formula (4.4), we have

$$
\lim _{n \rightarrow \infty} \int_{T_{n}} \Psi(w, z) d w=0, \quad z \in K ; \quad T_{n}=\left\{w=r_{n} e^{i \theta}: \theta \in[c, d]\right\}
$$

where the convergence is uniform in $z \in K$. Combining this with formula (4.4), by standard methods of complex analysis we obtain:

$$
\alpha(z)=\Phi(z)+\lim _{R \rightarrow \infty} \sum_{\left|b_{k}\right|<R} \underset{w=b_{k}}{\operatorname{res}} \Psi(w, z), z \in \mathbb{C} \backslash \Lambda_{g} ; \Phi=1 / g .
$$

Now by Theorem 1 and Proposition 1 we conclude that the function $\Phi=1 / \mathrm{g}$ can be expanded into partial fractions (see (2.1)).

2. Suppose that $B_{g}=\Gamma_{1}$. Consider the integral $\alpha_{n}(z)$ of the form (4.4), where

$$
L:=T_{n}=\left\{w=r_{n} e^{i \theta}: \theta \in(-\pi \pi]\right\}
$$

is the circumference of radius $r_{n}$ centered at the origin. We choose $\varepsilon \in(0, m)$, where $m=$ $\min \left\{h_{g}(\theta):|\theta| \leqslant \pi\right\}$. In this case formulae (4.7), (4.8) remain valid if we replace $[c, d]$ with 
$(-\pi \pi]$. By the same arguments we see that $\Phi=1 / g$ can be expanded into partial fractions. Moreover, for $\Phi$ in expansion (2.1) we have

$$
\alpha(z):=\lim _{n \rightarrow \infty} \alpha_{n}(z) \equiv 0, \quad z \in \mathbb{C} .
$$

Remark 1. In the notations of the first part of the proof by Property 2 we have that the generalized indicator $h_{g}(\theta)$ of $g$ is $\rho$-trigonometrical for $\theta \in(\gamma, a) \cup(b, \beta)$. Since $h_{g}$ is positive on these intervals, it is possible if $a-\gamma<\pi / \rho, \beta-b<\pi / \rho$. If $B_{g}=\Gamma_{1}$ (see part 2), then, arguing as above, we find $a+2 \pi-b<\pi / \rho$.

Remark 2. Let $M$ be any closed cone centered at the origin such that $M_{F} \subset M \subset M_{B}$, where $M_{F}, M_{B}$ are the cones centered at the origin and generated by the arcs $F_{g}, B_{g} \neq \Gamma_{1}$ (see 4.1), 4.2), respectively. Estimating the integral of the form (4.4), we obtained the following property of the entire function $\alpha(z)$ in the expansion of $\Phi=1 / \mathrm{g}$ into partial fractions:

$$
\alpha(z)=o(1), \quad z \rightarrow \infty, \quad z \in M .
$$

Remark 3. Expansion in partial fractions is possible for the reciprocal of some CRGfunction $g$ under the condition (see (4.1), (4.2))

$$
F_{g} \subset\left\{e^{i \theta} \in \Gamma_{1}: h_{g}(\theta)=0\right\}
$$

as the following well-known expansion shows.

Consider the popular entire function $g_{0}=\sin z$. It is of order 1 and normal type, and its indicator is equal to $|\sin \theta|, \theta \in \mathbb{R}$. The condition (4.9) is true for $g_{0}$ (except $z_{0}=0$ ). It is known that

$$
\csc z=\frac{1}{\sin z}=\frac{1}{z}+\lim _{m \rightarrow \infty} \sum_{k=1}^{m}(-1)^{k}\left[\frac{1}{z-k \pi}+\frac{1}{z+k \pi}\right], \quad z \neq k \pi, k \in \mathbb{N} .
$$

For the function $g_{1}(z)=z^{-1} \sin z$, (16) is valid, too. However, the following expansion

$$
\frac{z}{\sin z}=1+\lim _{m \rightarrow \infty} \sum_{k=1}^{m}(-1)^{k}\left[\left(1+\frac{\pi k}{z-k \pi}\right)+\left(1-\frac{\pi k}{z+k \pi}\right)\right], \quad z \neq k \pi, k \in \mathbb{N},
$$

holds true. This means that $P_{k}(z) \equiv(-1)^{k}, z \in \mathbb{C}, k \in \mathbb{Z} \backslash\{0\}$ in the expansion (1.2) for $1 / g_{1}$. 2 .

By the proof (see part 2) we find the following generalization for some statements of Theorem

Corollary 1. Let in the notation of Theorem $1 g \in A[\rho(r)]$, and $F_{g} \subset B_{g}=\Gamma_{1}$ (see (4.1), (4.2)). If the assertion of Property 1 is valid for the generalized indicator $h_{g}$ of $g$ (see (3.2)), then the function $\Phi=1 / \mathrm{g}$ expands in partial fractions. Moreover, $\alpha(z) \equiv 0$ in the expansion (2.1) for $\Phi$.

\section{The CASE OF ERG-FunCtions With ZEROES ON A RAY.}

We present some conditions of expansion in partial fractions for reciprocals of ERG-functions of finite order $\rho>0$ with zeroes on a ray. By Property 2, the generalized indicator of each such function is $\rho$-trigonometrical one. Without loss of generality it is sufficient to consider ERG-functions with negative zeroes.

Let

$$
g(z)=\prod_{k=1}^{\infty} E\left(z / b_{k} ; p\right)
$$

be the Weierstrass canonical product of genus $p$, where

$$
0>b_{1}>b_{2}>\ldots>b_{n}>\ldots, \quad\left|b_{n}\right| \rightarrow \infty
$$


is the sequence of zeroes of $g$. We denote by $n(r)$ the sum of orders of the zeroes of $g$ in the disk $K(r)=\{z \in \mathbb{C}:|z| \leqslant r\}$. Assume that $n(r)$ is a function of finite order $\rho>0$ and of normal type with respect to proximate order $\rho(r), \rho(r) \rightarrow \rho$, and there exists the limit

$$
\lim _{r \rightarrow \infty} \frac{n(r)}{r^{\rho(r)}}=\Delta, \quad \Delta \in(0, \infty) .
$$

Suppose $g \in A[\rho(r)]$ is an ERG-function. We recall some known properties of $g$.

Theorem A ([11, Ch. 1,2,3]). Let in the above notation (see (5.1) ) $p<\rho<p+1$. Then $g \in A[\rho(r)]$ is an ERG-function, and the generalized indicator of $g$ is defined by the formula

$$
h_{g}(\theta)=A_{g} \cos \rho \theta, \quad|\theta| \leqslant \pi, \quad A_{g}=\frac{\pi \Delta}{\sin \pi \rho} .
$$

Theorem B ([4, Ch. 2], [11, Ch. 2,3]). Let in the same notation $\rho \in \mathbb{N}$.

1. If $p=\rho-1$, then $g \in A\left[\rho_{-}(r)\right]$ is an ERG-function, where $\rho_{-}(r)$ is the proximate order such that

$$
r^{\rho_{-}(r)}=r^{\rho} L_{-}(r), \quad L_{-}(r)=\int_{r}^{\infty} t^{\rho(t)-\rho-1} d t \downarrow 0 \text { as } r \rightarrow \infty .
$$

Moreover, the generalized indicator of $g$ is defined by the formula (see (5.1))

$$
h_{g}(\theta)=-\Delta \cos \rho(\theta+\pi), \quad|\theta| \leqslant \pi .
$$

2. If $p=\rho$, then $g \in A\left[\rho_{+}(r)\right]$ is an ERG-function, where $\rho_{+}(r)$ is the proximate order such that

$$
r^{\rho_{+}(r)}=r^{\rho} L_{+}(r), \quad L_{+}(r)=\int_{1}^{r} t^{\rho(t)-\rho-1} d t \uparrow \infty \text { as } r \rightarrow \infty .
$$

Moreover, formula (5.3) remains true with the only difference that the minus sign on the right in (5.3) should be omitted.

Remark 4. A.A. Goldberg [14] proved the inverse assertion in the case $\rho \in \mathbb{N}$.

Let $\rho(r)$ be a proximate order. Suppose $\rho(r) \rightarrow \rho \in \mathbb{N} ; L(r)=r^{\rho(r)-\rho} ; \Delta \in(0, \infty)$. If $L(r) \downarrow 0, r \rightarrow \infty$ then there exists a proximate order $\rho_{1}(r) \rightarrow \rho$ and the Weierstrass canonical product $g$ of genus $p=\rho-1$ and of order $\rho(r)$ with negative zeroes satisfying the condition $n(r) / r^{\rho_{1}(r)} \rightarrow \Delta$ as $r \rightarrow \infty$ (cf. (5.1) ). Moreover $g$ is the ERG-function such that its generalized indicator is defined by formula (5.3). A similar statement remains true in the case $L(r) \uparrow$ $\infty, r \rightarrow \infty$ (see Statement 2 in Theorem B).

We denote by $B[\rho(r)]=\{g\}$, where $\rho(r) \rightarrow \rho>0, r \rightarrow \infty$ is a proximate order, the class of Weierstrass canonical products satisfying the equality of the form (5.1). By formulae (5.2), (5.3) and Theorem 2 we obtain the following condition of expansion into partial fractions for the reciprocals of functions of this class.

Theorem 3. Let $g \in B[\rho(r)]$. The function $1 / g$ expands in partial fractions,

1) if $\rho \in \bigcup_{k=0}^{\infty}(k, k+1 / 2)$;

2) if $\rho \in \mathbb{N}$ and $g$ is a function of order $\rho$ and of maximal type.

In the case $\rho=1$ Theorem 3 was proved in [10, Thm. 6.5].

\section{The CASE OF THE MORE COMPLICATED MEROMORPHIC FUNCTION.}

Here we study the case of a meromorphic function $\Phi(z)=f(z) / g(z)$, where $f, g$ are entire functions of finite order without common zeroes. In this case some generalizations of Theorem 2 and Corollary 1 are true. It is possible to prove them by the same way. For simplicity we restricted ourselves by the case of Corollary 1. 
Definition 3. Let $\rho_{f}(r), \rho_{g}(r)$ be proximate orders of the functions $f, g$, respectively. The function $f$ is said to grow slower than $g$ if either $r^{\rho_{f}(r)}=o\left(r^{\rho_{g}(r)}\right)$ as $r \rightarrow \infty$ and $h_{g}(\theta)>$ $0, \forall \theta \in \mathbb{R}$ or $r^{\rho_{f}(r)}$ is equivalent to $r^{\rho_{g}(r)}$ as $r \rightarrow \infty$, but $h_{f}(\theta)<h_{g}(\theta), \forall \theta \in \mathbb{R}$, where $h_{f}, h_{g}$ are the generalized indicators of $f$ and $g$, respectively.

Theorem 4. Assume that function $f$ grows slower than $g$, and the assertion of Property 1 is valid for the generalized indicator $h_{g}$ of $g$ (see (3.2)). Then the function $\Phi=f / g$ expands in partial fractions. Moreover, $\alpha(z) \equiv 0$ in the expansion (2.1) for $\Phi$.

\section{Conclusion.}

The author is grateful to A.G. Malytin and M.L. Sodin for a useful discussion of some assertions of this paper. In conclusion I would like to share my impressions, reminiscences of I.F. Krasichkov-Ternovsky, to his respectful memory I dedicate this paper.

I met Igor Fedorovich in 1971, while participating the conference on the number theory in Kharkov. He impressed me by a large scale of his studies, an extraordinary erudition, and on the other hand, by his humor, amicability, simple communication with other mathematicians. When I opened his published papers, I understood that the first impressions on new results presented during his talks on mathematical forums is just an superelevation of an "iceberg", as he was called by Ukrainian mathematician V.A. Takchenko, since his works stunned by the deep penetration into the matter of a studied problem. For me personally, he was a good friend, alive etalon of an outstanding mathematician. During our meetings in various conferences or during my trips to Moscow or to Ufa, where he was in different time, I tried to "synchronize the watches", to known his opinion whether the problems I worked on are interesting and not "casual".

The results of this work were presented in 2001 in the congress in Kharkov dedicated to the century anniversary of N.I. Akhiezer. We lived in the same room with Igor Fedorovich. The subject of my talk happened to be close to his previous studies. I listened to a whole lecture on the directions in which this problem is to be developed (unfortunately, I was not so familiar with them). His suggestion was interesting for me, we initiated a communication, but, unfortunately, it was stopped due to various reasons. Our next meeting was in 2007 in a big conference in Ufa dedicated to the memory of A.F. Leontiev and this meeting was the last. At that time, the healt of Igor Fedorovich did not allow to speak seriously on the mathematics. Now I decided to publish the full text of the paper in its original version hoping that the mathematicians will find the developing of its results meriting a blessed memory of Igor Fedorovich Krasichkov-Ternovskii.

\section{REFERENCES}

1. A.I. Markushevich. Theory of analytic functions. V. 2. Nauka, Moscow (1968). (in Russian).

2. L.S. Maergoiz. On partial fraction expansion for meromorphic functions// Mat. Fiz. Anal. Geom. 9:3, 487-492 (2002).

3. B.Y. Levin (in collaboration with Yu. Lyubarskii, M. Sodin, V. Tkachenko). Lectures on entire functions. (Rev. Edition). AMS, Providence, Rhode Island (1996).

4. A.A. Gol'dberg, I.V. Ostrovskii. Value distribution of meromorphic functions. Nauka, Moscow (1970). (in Russian).

5. I.V. Ostrovskii On a class of entire functions // Dokl. Akad. Nauk SSSR. 229:1, 39-41 (1976). [Soviet Math. Dokl. 17:8-9, 977-981 (1976).]

6. A.A. Gol'dberg. Meromorphic functions // Itogi Nauki i Tekhn. Ser. Mat. Anal. 10, 5-79 (1973). [J. Soviet Math. 4:2, 157-216 (1975).]

7. J. Clunie, A. Eremenko and J. Rossi. On equilibrium points of logarithmic and Newtonian potentials// J. London Math. Soc. 47:2, 309-320 (1993). 
8. V.B. Sherstyukov. Representation of the reciprocal of an entire function by series of partial fractions and exponential approximation // Matem. Sborn. 200:3, 147-160 (2009). [Sb. Math. 200:3, 455-469 (2009).]

9. V.B. Sherstyukov. Expanding the reciprocal of an entire function with zeroes in a strip in a Krein series // Matem. Sborn. 202:12, 137-156 (2011). [Sb. Math. 202:12, 1853-1871 (2011).]

10. L.S. Maergoiz. The indicator diagram of an entire function of proximate order and its generalized Borel-Laplace transforms // Alg. Anal. 12:2, 1-63 (2000). [St. Petersburg Math. J. 12:2, 191-232 (2001).]

11. B.Ya. Levin. Distribution of the zeros of entire functions. Fizmatgiz, Moscow (1956). [Amer. Math. Soc. Providence, R.I. (1964).]

12. Azarin V. Growth theory of subharmonic functons. Birkhäuser Advanced Texts. Birkhäuser, BaselBoston-Berlin (2009).

13. Brelot M. Éléments de la théorie classique du potentiel. Centre de documentation universitaire, Paris (1961).

14. Gol'dberg A.A. Integral representation of slowly varying monotonic functions // Izv. Vyssh. Uchebn. Zaved. Mat. 4, 21-27 (1988). [Soviet Math. (Izv. VUZ. Matem.) 32:4, 27-35 (1988).]

Lev Sergeevich Maergoiz,

Siberian Federal University,

Svobodnii av., 79,

660041, Krasnoyarsk, Russia

E-mail: bear.lion@mail.ru 